\title{
Stem cells: disease models that show and tell
}

\author{
Vivien Marx \\ Combining gene-editing techniques with induced pluripotent stem cells is both powerful and tricky.
}

The cells looked beautiful-and that was bad news, recalls Clive Svendsen, a stem cell researcher and neurobiologist at CedarsSinai Medical Center. He was developing a cell-based disease model for spinal muscular atrophy (SMA). It's a heartbreaking, untreatable disease. Infants with SMA become paralyzed when they are around six months old, and they die before their second birthday.

At the time, Svendsen was at the University of Wisconsin-Madison working with James Thomson on induced pluripotent stem cells (known as iPS cells or iPSCs), which are made by reprogramming donor cells to an embryo-like state and which can be differentiated in the lab into any number of cell types ${ }^{1}$. In this case, the researchers had transformed iPSCs from an infant with SMA and from his healthy mother into motor neurons. They wanted to create a disease-in-a-dish, an in vitro model for SMA. But the neurons from mother and son looked identical.

Svendsen wondered if the six-week-old cells might be too young to show the dis-

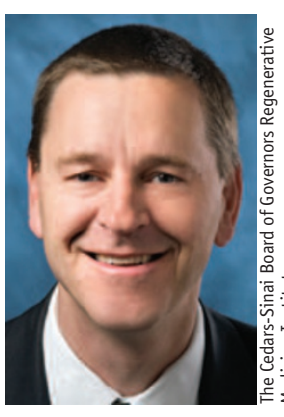

Clive Svendsen hopes gene-edited iPSCs can become full models of neurodegenerative diseases. ease, not unlike cells in a person prior to disease onset. He and his team generated motor neurons, plated the cells and let them develop for ten weeks. There was a huge phenotypic difference: the mother's neurons were thriving, whereas around $70 \%$ of the baby's neurons had begun

to $\mathrm{die}^{2}$. "It was one of those moments - and you don't have many of these moments in science careers - when you go, 'Wow,' says Svendsen.
Now at Cedars-Sinai, Svendsen continues to work with iPSCs to model SMA and neurodegenerative diseases such as amyotrophic lateral sclerosis (ALS). When looking at a familial form of ALS with iPSC-derived neurons, he and his collaborators noticed that the cells again lacked large phenotypic differences. That may not be surprising, he says, given that ALS, too, is a late-onset disease: the cells are from patients who had led active tennisand rugby-playing lives until falling ill in their 50 s. Only around $10 \%$ of patients have the familial form of ALS; the other cases are sporadic. Both categories are due to, as of yet, mainly unknown molecular mechanisms. His team and others found one molecular clue in familial ALS: they saw indications of RNA foci in the diseased cells. These foci are expanded RNA repeats that lead to flawed transcription and protein production, which could play a role in ALS.

Researchers such as Svendsen are now combining iPSCs and gene editing, a technique that allows targeted changes to be made at a chosen genomic location. Equipped with this double tool set, they want to model disease in new ways ${ }^{3,4}$. Gene-edited iPSCs can hopefully prove their predictive mettle in basic research and in therapy development. They will enhance understanding of healthy cells, too: new models can do better justice to the cell's dynamic changes in space and time, says Rick Horwitz, director of the Allen Institute for Cell Science.

\section{Editing genes to understand disease}

Many stem cell researchers doing gene editing began with zinc-finger nucleases (ZFNs), moved on to transcription activator-like effector nucleases (TALENs) and now frequently use the clustered, regularly interspaced, short palindromic repeats (CRISPR) system with the nuclease Cas9.

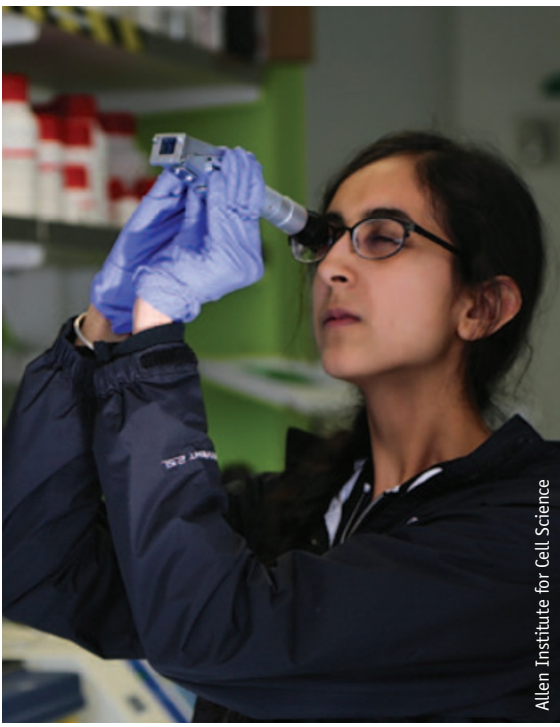

Gene-editing and iPSCs are a double tool set for modeling disease in new ways.

With gene editing, researchers can generate isogenic iPSC lines: two sets of cells that have identical genomes except for the edited change. "These are the optimal controls for our disease-modeling studies since they differ from the diseased iPSCderived heart cells only in the gene of interest, while having an identical genetic background," says stem cell researcher Lior Gepstein at the Technion. Gepstein uses patient and disease-specific iPSCderived heart cells to model cardiovascular disease and also for drug testing (Box 1).

In the lab he is just setting up, stem cell biologist Jared Sterneckert at Technische Universität Dresden uses gene-edited iPSCs to model neurodegenerative diseases-Parkinson's in particular, for which good animal models are lacking. Researchers have yet to find the molecular mechanisms underlying this disease. But gene editing of cells derived from people with familial Parkinson's indicate the 


\section{BOX 1 SCREENING DRUGS WITH GENE-EDITED IPSCS}

Cells derived from gene-edited iPSCs can also help test efficacy and toxicity of compounds ${ }^{6}$. As Joseph Wu explains, gene-edited iPSCs can serve academics as their own drug-screening panel. For example, it can be hard for labs to recruit diverse populations of patients with differing mutations as donors. Studies risk grinding to a halt if the cohort lacks the mutations of interest. "The genome-editing approach provides a nice alternative to this dilemma," he says.

But piquing industry interest in a potentially useful compound takes insight into mechanism and scaled-up, consistent results, says Jared Sterneckert. An academic might run gene-edited iPSC experiments in triplicate wells, do a $t$-test and call results 'significant', but, he says, industry wants significance in thousands of samples.

The main reason why drugs are taken off the market, says Lior Gepstein, is due to their propensity to generate life-threatening heart arrhythmias. The mechanism is most commonly that a drug blocks a kind of potassium channel. This prolongs the duration of the QT interval, which is when the heart contracts and is refilled with blood before its next contraction. But a lack of human cardiac models limits the early detection of such side effects. Large-scale screening of cardiomyocytes derived from iPSCs that have a variety of genetic backgrounds, both patient derived and obtained through gene editing, could be uniquely valuable, he says.

power of these tools to help decipher and model complex disease.

While still at the Max Planck Institute for Molecular Biomedicine, Sterneckert was part of the team that used gene-edited iPSCs to correct a mutation called G2019S in leucine-rich repeat kinase 2 (LRRK2) that is linked to a familial form of Parkinson's disease. Editing the mutation reversed disease phenotypes, such as reduced outgrowth of branches called neurites in neurons derived from these cells ${ }^{5}$.

Depending on their questions, scientists might start with cells derived from healthy or diseased donors. They may use geneediting techniques to reverse known mutations in the cells, introduce new mutations or introduce fluorescent markers as reporters or as fusions to particular genes. In Gepstein's view, adding mutations to iPSCs derived from healthy donors can help create new disease models and let researchers evaluate the role of different genes in a planned manner, he says. They can see whether modifier genes and polymorphisms increase or decrease the risk of acquiring the disease phenotype and better understand patientto-patient variability.

Starting with a disease cell line and then correcting the mutation allows one to ask whether the mutation is necessary for a given disease because, under these conditions, the cell has a genetic background "permissive" for the disease, says Kiran Musunuru, a cardiologist and stem cell researcher at Harvard University and Brigham and Women's Hospital. Starting with a wild-type cell line requires that the mutation in question be sufficient to cause disease and that the mutation can lead to a distinct phenotype in the gene-edited cell line. Researchers can also introduce a series of mutations into a wildtype cell line and make an array of matched cell lines, which all have the same genetic background, each with a different mutation. "Then you are really comparing apples to apples," he says.

As Kang Zhang, a stem cell biologist and physician at the University of California at San Diego also points out, it is not always easy to be sure that a mutation made in the genome of iPSCs from healthy donors is relevant to the disease, especially if a particular genotype does not lead to a clear phenotype.

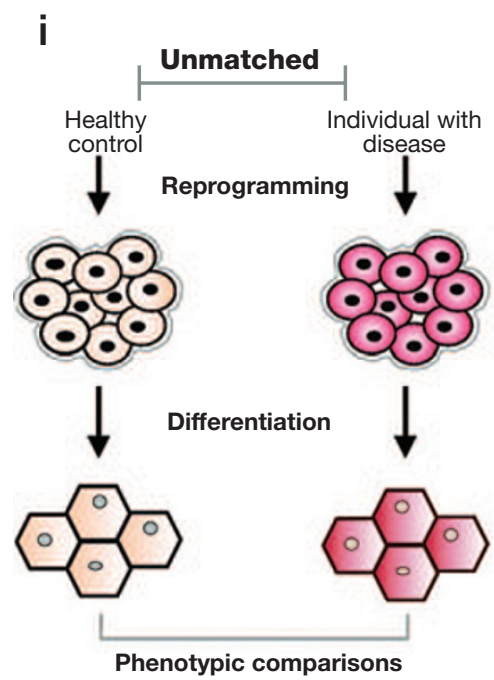

Zhang applies gene-editing techniques to model aging processes as well as specific eye disorders such as macular degeneration. Gene editing accelerates work in iPSCs and animals, and, he says, "in either case, it is much faster and cheaper when compared to traditional technologies." Zhang's work is part of the $\$ 32.3$ million initiative by the California Institute for Regenerative Medicine, the state's stem cell agency, to set up a bank of 9,000 stem cell lines from 3,000 individuals and representing 11 diseases. The teams are using tissue samples from people with these diseases and converting cells into iPSCs.

Both experimental designs-starting with cells from patients with disease or editing cells from healthy donors-are helpful and deliver complementary information such as when assessing the impact of gain or loss of function related to a mutation, says Joseph $\mathrm{Wu}$, a stem cell researcher and cardiologist at Stanford University School of Medicine.

At Boston University, Darrell Kotton and colleagues are studying lung disease, and the lung tissue's ability to regenerate after injury, by editing iPSCs derived from both healthy and diseased donors. These strategies give different views of the perturbed gene locus, says Kotton, and "our philosophy is to pursue both."

Patient-derived cell lines are valuable for incompletely penetrant genetic diseases, in which not all carriers of a mutation have the disease. In these patients, everything matters: the mutation of interest, disease-modifying genes and the entire genetic background. This background is "likely to be particularly important for establishing a faithful and

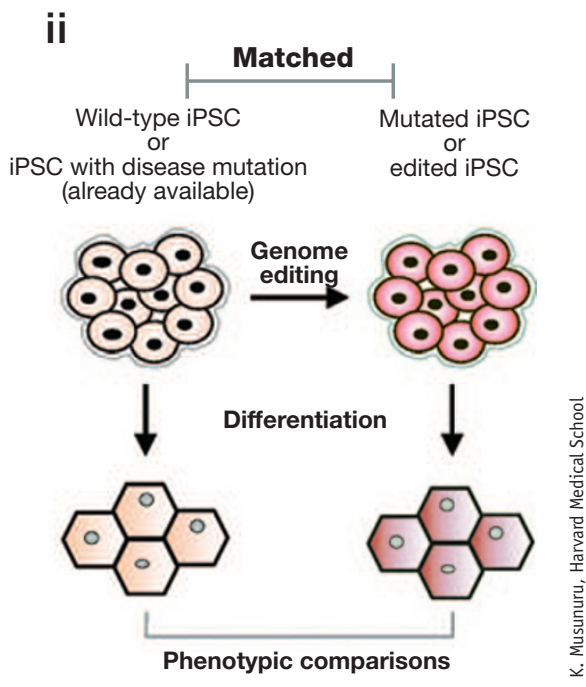

Two possible study designs: (i) iPSCs are derived from individuals with disease and healthy donors; (ii) matched cell lines are created when the genome of pluripotent stem cells is edited. 
robust in vitro disease model," says Kotton. "Another part of the story," says Musunuru, "will be environmental influences, which will be difficult to study in iPS cells in vitro."

Similarly, some copy-number variations and polymorphisms associated with certain psychiatric conditions can be phenotypically silent in some cases, says Alexandra Benchoua, a stem cell biologist at I-Stem, a French research institute funded by the government and charities. She uses iPSCs and gene editing to study neuropsychiatric disorders.

Monogenic diseases tend to be caused by mutations that are sufficient to cause a disease. Over the last year, gene-edited iPSCs have been used to model hypertrophic cardiomyopathy, a classic Mendelian disease, and Barth's syndrome, a mitochondrial disease. In polygenic and more complex diseases, mutation combinations play a role; individual mutations are not sufficient to cause the disease even though they might, in some cases, be necessary for it.

"Because of the nature of complex disorders, I think it will be much harder to study them using iPS cells," says Musunuru. He battles such challenges as he tries to model myocardial infarction genes with iPSCs.

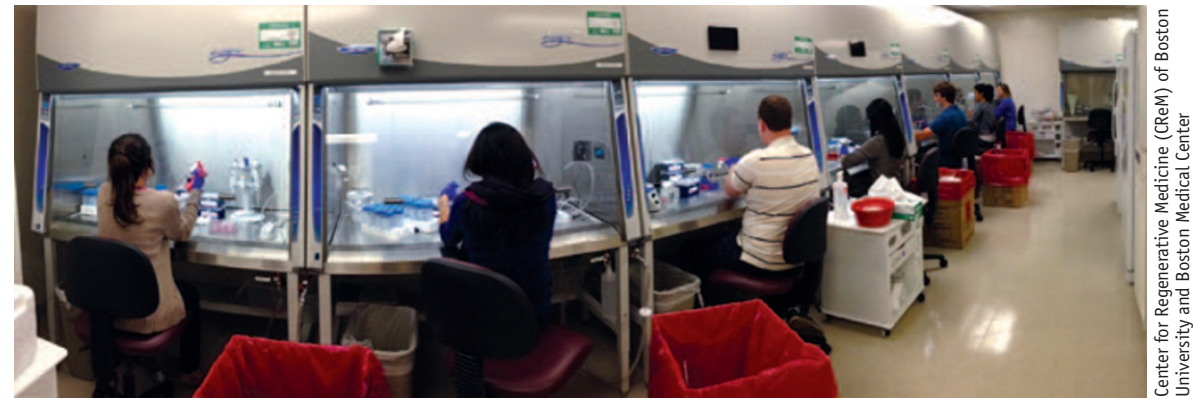

Building libraries of edited stem cells is a scaled-up lab enterprise.

Given the multitude of disease-shaping influences, he says his experience is perhaps not surprising.

\section{Facing challenges}

Gene editing lets scientists create isogenic iPSC lines that help to minimize variability when comparing cells derived from diseased donors with those from unmatched, unrelated healthy donors. But experimenters must still address other sources of variability.

Just as physicians see diverse symptoms in patients with the same disease, says Sterneckert, researchers see diversity in differentiated stem cells from donors with the same disease. Neurons derived from iPSCs can vary in gene expression, morphology and behavior, all of which can make it hard to see differences between disease and wildtype cells. Even when researchers create isogenic cell lines, cells can still differ in ways that confound pinpointing the effect of specific mutations. Differentiation in vitro can vary between cell lines, for example. As scientists generate, expand and passage stem cells, genetic changes such as singlenucleotide variants or rearrangements can occur, says Musunuru. Also, iPSCs can vary in terms of their epigenetic states, which can affect differentiation.

When working with iPSCs and gene editing, researchers must keep in mind that they

\section{BOX 2 SOME STEM CELL LIBRARIES}

Gene-edited iPSC libraries can help to sort out the many sources of diversity in iPSCs and their derivatives. There can be a whole range of traits of what is considered normal and diseased. Results might differ, for example, if one compares gene-edited iPSCs from different labs that have been generated with varying differentiation protocols, says Peter Reinhardt of the Technische Universität Dresden. With an isogenic pair of edited and unedited iPSCs on hand from a library, scientists will have a better sense of the phenotypes they might expect in their own experiments.

A library should ideally be geared toward sharing, says Joseph Wu. It should be broad and deep: broad enough to cover a diverse panel of different diseases, and deep enough to have several types of mutations for each disease. That will help researchers to correlate genetic variants with phenotypic changes when using iPSCs.

Allen Institute for Cell Science. The newly founded institute is devoted to building a predictive model of the cell and enabling systems approaches to cell biology. A team of scientists will use CRISPR-based techniques to build a library of iPSCs with major molecular machines and organelles tagged with green fluorescent reporters.

Boston University. Here, stem cell biologist Darrell Kotton and his team have built a human and mouse iPSC bank that includes cell lines derived from patients with lung diseases. Now the team is adding gene-edited lines that carry fluorescent reporter genes. They use the range of gene-editing tools, says Kotton: lentiviruses, ZFNs, TALENs and CRISPR-Cas.

Cedars-Sinai Medical Center. Cedars-Sinai has a library of iPSCs derived from patients with many types of neurodegenerative disorders. With funds from the ALS Association's 'Ice Bucket Challenge', the lab of Clive Svendsen is generating iPSCs from ALS patients. Several Cedars-Sinai labs are also using gene-editing techniques to expand this library.

Coriell Institute for Medical Research. Coriell has a repository, funded by the US National Institute of Neurological Disorders and Stroke, with iPSCs for neuroscience research, which can include gene editing.

\section{California Institute for Regenerative Medicine. The state's} stem cell agency is creating a bank of 9,000 stem cell lines from 3,000 individuals, representing 11 diseases.

\section{Stanford Cardiovascular Institute iPSC Biobank. Stanford is} creating 1,000 iPSC lines from patients of different ethnicities, genders and ages who suffer from various types of cardiovascular disease. The plan is also to include numerous genome-edited cell lines relevant to the disease.

StemBANCC. A European academic-industry consortium is building an iPSC bank with cells from 500 donors from which 1,500 cell lines will be derived; the cells will be characterized also for use in drug development. The emphasis is on pain research, neurological disorders and diabetes. 


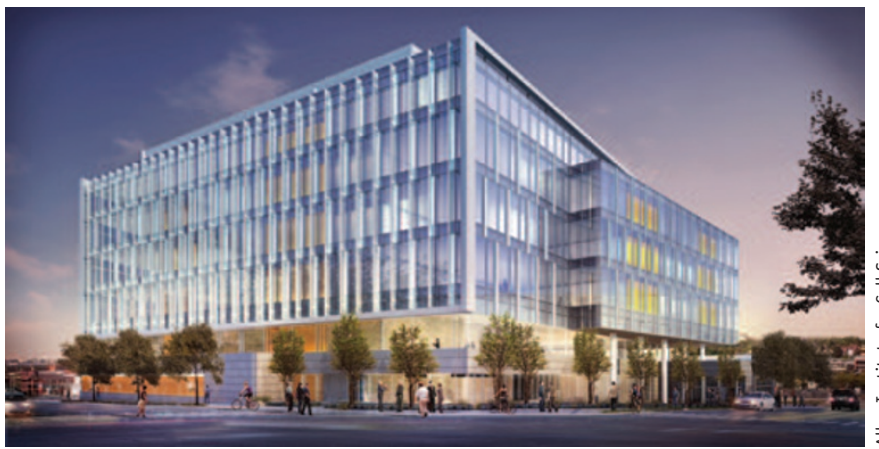

Slated for completion this fall, a new building will house two Allen Institutes; one focuses on brain science, the other on cell science. biomarker for Parkinson's disease, aggregate in healthy and diseased neurons.

A number of libraries are devoted to gene-edited stem cells (Box 2). Using gene editing to introduce markers helps to build libraries and study many functions in iPSCs, says Musunuru. For

need adequate controls, says Musunuru. Studying one or several iPSC lines from a patient with a genetic disorder without one or several properly matched controls is not informative. "Studying four edited cell clones and four control clones is much more robust than studying one and one," he says.

Other challenges relate to the gene-editing process itself. Gene edits can occur off-target, resulting in genomic changes other than the targeted one. To thwart these issues, says Musunuru, scientists can generate a group of edited cell clones using different CRISPR guide RNAs or TALEN or ZFN pairs. Offtarget changes may still occur, but they will likely be dissimilar between guide RNAs or technologies, whereas the on-target mutations will be the same.

Making gene edits in stem cells can be nontrivial, especially when a new section of DNA is inserted via homologous recombination, says Svendsen. CRISPR has relatively low efficiency in these cells, he says. A researcher might look at 100 gene-edited clones, but only 3 will have the correct gene inserted.

Stem cells are also not readily transfected, says Sterneckert. And separately, when it comes to expression, scientists need to find vectors and promoter systems that the cells will express and not silence, he says.

Finally, it remains a substantial challenge to reproduce disease-relevant scenarios in a Petri dish. Cells in a plate do not model all of physiology, for example, drug-induced inflammation, says Svendsen. Furthermore, cells are naturally stressed just by the fact that they are outside of the body, he says.

A number of labs are editing iPSCs to generate fluorophore-tagged reporter genes. For instance, Sterneckert and his team want to create gene-edited cells tagged with fluorescent reporters to better study where specific proteins such as $\alpha$-synuclein, a potential example, tagging many cytoskeletal proteins with fluorescent proteins helps to reveal cell structure in iPSC-derived cells in real time, he says, in both differentiating and differentiated cells.

\section{Building big libraries} remit of the Allen Institute for Cell Science, newly launched with a $\$ 100$ million donation from Microsoft cofounder and philanthropist Paul Allen. A new building, slated to house both this institute and the Allen Institute for Brain Science, will be ready in the fall.

A first project is to build a library of characterized iPSCs from healthy donors and into which green fluorescent protein tags will be edited with CRISPR, says Horwitz, the institute's director. The library will be generated on an industrial scale, which is the kind of work that teams of researchers can do and that individual labs cannot readily do on their own.

The tags will mark all of the cell's major molecular machines and organelles. The Allen Institute team would like the research community to advise on the best labels and where to place them, he says, such as the five brightest labels for endosomes that don't perturb function. "That's where the community is going to be involved, helping us to choose exactly what to do," says Horwitz. The cells will be available to academic and industry scientists.

Once this library is established, work will continue to perturb these cells, such as by changing the environment or by using gene-editing techniques to make mutations or to knock genes in or out. By altering the function of specific molecular machines, the Allen Institute researchers can then track how an effect propagates through the iPSC's entire system and study many
Libraries benefit from scale, which is the cellular activities at once. For example, they might track mitochondria in a dividing cell, determine their locations and monitor their changing activities. "We want to look at the cell as a system," says Horwitz.

Around 75 scientists will be hired, and the long-term goal at this Allen Institute is to create a visual, animated model of the cell that does justice to the cell's changes and its variability. "Everything is dynamic in space and time," says Horwitz.

The cells in the iPSC library will be differentiated into cardiomyocytes because of their disease relevance and robust differentiation protocols, he says. Another set will be differentiated into epithelial cells. The cells will be characterized in plates as well as in threedimensional cell culture, both of which are challenging for the planned imaging pipelines. Many cell movies will be captured. But scientists cannot just watch movies all day, says Horwitz.

These captured data will be used to develop predictive models of the cell. The models will be based on experimental information collected across spatial and temporal scales, says Horwitz. It will address the typical variation between iPSCs and

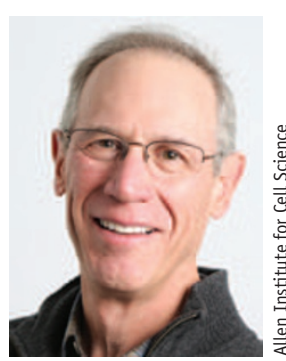

"We want to look at the cell as a system," says Rick Horwitz. the cells derived from them. These models even offer the specter of computational gene editing. "If you perturb this gene, we predict this will happen in the cell," he says. "That's aspirational, but that's our goal."

Scientists have already clocked successes with gene-edited iPSCs. It will still take time to see whether this approach delivers the robust in vitro disease models they seek. Large libraries of edited human cells will help researchers to more systematically tackle the many technical and biological sources of variation in using iPSCs and their derivatives to study human disease.

1. Takahashi, K. \& Yamanaka, S. Cell 126, 663-676 (2006).

2. Ebert, A.D. et al. Nature 457, 277-280 (2009).

3. Sterneckert, J.L., Reinhardt, P. \& Schöler, H.R. Nat. Rev. Genet. 15, 625-639 (2014).

4. Musunuru, K. Dis. Model. Mech. 6, 896-904 (2013).

5. Reinhardt, P. et al. Cell Stem Cell 12, 354-367 (2013). 6. Wang, Y. et al. J. Am. Coll. Cardiol. 64, 451-459 (2014).

Vivien Marx is technology editor for Nature and Nature Methods

(v.marx@us.nature.com). 